\title{
Solar-Like Oscillations of Procyon A: Stellar Models and Time Series Simulations versus Observations ${ }^{1}$
}

\author{
C. Barban, E. Michel \\ Observatoire de Paris, DASGAL, CNRS UMR 8633, 92195 Meudon, \\ France
}

M. Martic, J. Schmitt, J. C. Lebrun

Service d'Aéronomie, CNRS, 91371 Verrières le Buisson, France

\author{
A. Baglin \\ Observatoire de Paris, DESPA, CNRS UMR 8632, 92195 Meudon, \\ France \\ J. L. Bertaux \\ Service d'Aéronomie, CNRS, 91371 Verrières le Buisson, France
}

\begin{abstract}
The aim of this paper (further developed in Barban et al. 1999) is to present new evidence of the possible stellar origin of the observed excess power in the power spectrum of Procyon A presented in Martic et al. (1999) by comparing these observational data with theoretical predictions and numerical simulations.
\end{abstract}

Stellar models are calculated for Procyon A with the CESAM evolutionary code (Morel 1997). We use input physics appropriate to the revised astrometric mass $(1.46 \pm 0.04) M_{\odot}$ found by Girard et al. (Girard 1998). For these models, we compute the expected oscillation spectrum for $\ell=0,1,2$ modes including $m \neq 0$, according to theoretical amplitude predictions (Houdek et al. 1999), and with oscillation frequencies calculated with the adiabatic oscillation code of Christensen-Dalsgaard (Christensen-Dalsgaard \& Berthomieu (1991) (Figure 1a). Time series are then simulated, under the same conditions as the observations, and compared by Fourier analysis (Figure 1b) with the observed ones (Figure 1c).

The observed frequency range of the observed excess power, i.e. $0.5-1.5$ $\mathrm{mHz}$, is in perfect agreement with the predicted one. The irregular shape of this excess power can be explained by interference between modes through the observational window. Equidistant peaks are found at approximately $56 \mu \mathrm{Hz}$; this value corresponds to the expected large separation of Procyon A (Barban et al. 1999). We conclude that the observed excess power has a stellar origin.

\footnotetext{
${ }^{1}$ Based on observations collected at the Observatoire de Haute-Provence (CNRS, France)
} 


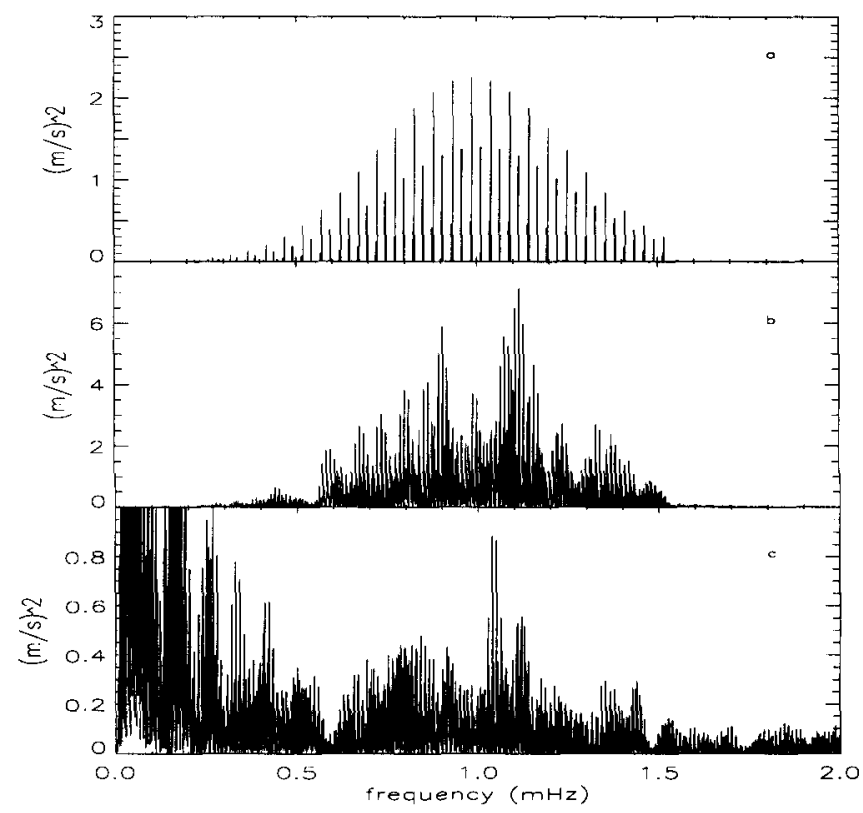

Figure 1. a: Theoretical oscillation spectrum corresponding to a model of $1.50 M_{\odot}$ at $2030 \mathrm{Myr}$. The highest peaks correspond to $\ell=0$, the intermediate ones to $\ell=1$ and the smallest ones to $\ell=2$. $\mathrm{b}$ : The previous oscillation spectrum including the 1998 November observational window. c: the power spectrum obtained for Procyon $A$ in 1998 November for the seven best nights merged together $(39.83 \mathrm{hr}$ of data).

\section{References}

Barban, C., Michel, E., Martic, M., Schmitt, J., Lebrun, J. C., Baglin, A., \& Bertaux, J. L. 1999, A\&A, 350, 617

Christensen-Dalsgaard, J. \& Berthomieu, G. 1991, in Solar Interior and Atmosphere (Tucson: University of Arizona Press), pp. 401-478

Girard, T. M. 1998, private communication

Houdek, G., Balmforth, N. J., Christensen-Dalsgaard J., \& Gough D. O. 1999, A\&A, in press

Martic, M., Schmitt, J., Lebrun, J.-C., Barban, C., Connes, P., Bouchy, F., Michel, E., Baglin, A., Appourchaux, T., \& Bertaux, J.-L. 1999, A\&A, in press

Morel, P. 1997, A\&AS, 124, 597 